\title{
Zinc Oxide Nanoparticles to Fight the COVID-19
}

\section{Mohammad Faizan ${ }^{1}$, Fangyuan Yu${ }^{1 *}$, Vishnu D Rajput ${ }^{2}$, Tatiana Minkina ${ }^{2}$ and Shamsul Hayat ${ }^{3}$}

${ }^{1}$ Collaborative Innovation Center of Sustainable Forestry in Southern China, College of

Forest Science, Nanjing Forestry University, Nanjing-210037, China

${ }^{2}$ Academy of Biology and Biotechnology, Southern Federal University, Rostov-on-Don,

Russia

${ }^{3}$ Plant Physiology Lab, Department of Botany, Aligarh Muslim University, Aligarh-202002,

India

*Corresponding Author: Fangyuan Yu, Collaborative Innovation Center of Sustainable

Forestry in Southern China, College of Forest Science, Nanjing Forestry University,

Nanjing-210037, China. E-mail: fyyu@njfu.edu.cn
Received: May 27, 2021

Published: June 09, 2021

(c) All rights are reserved by Fangyuan Yu., et

al.

\begin{abstract}
The pandemic of novel coronavirus disease (COVID-19) pandemic represents an unpredicted health concern and declared as 'public-health emergency of international concern" by the World Health Organization on 30 January 2020. The virus that is responsible for COVID-19 is known as Severe Acute Respiratory Syndrome Coronavirus 2 (SARS-CoV-2) and infected more than 160 million peoples as well as caused approximately 3.4 million deaths worldwide. Recent advancement in nanotechnology plays an important role in diagnosing the drug development programs. Zinc oxide nanoparticles (ZnO-NPs) have integrated into prevention of COVID-19. This review covers the use of NPs for the prevention of COVID-19 by several ways. The tools for diagnosis of coronavirus, nano-based vaccines and NPs as a drug delivery agent for the handling of virus infection have also been discussed.
\end{abstract}

Keywords: Diagnosis; Nanotechnology; Pandemic; Vaccines; Virus

\section{Introduction}

The first identified case of stern illness in humans due to coronavirus was reported in 2003 in China which resulted in the outbreak of Severe Acute Respiratory Syndrome (SARS) pandemic $[1,2]$. In 2012, the second outbreak of coronavirus reported in Saudi Arabia principal to the Middle East Respiratory Syndrome (MERS) epidemic [2,3]. Recently, the third eruption of this disease was occurred in Wuhan, China, in December 2019 and named as SARS-CoV-2. Based on the confirmed cases of coronavirus, on 11 February 2020 the official name 'COVID 19' was announced for the novel $\mathrm{CoV}$ variety that infected and shattered the lower respiratory tracts of a person suffering from Coronavirus in Wuhan, China [1].

Coronaviruses are a broad cluster of viruses belonging to the Coronaviridae family [1]. They are accounted to origin infection in humans and some animals. These viruses can be recognized by the occurrence of crown-like spikes on their surface [4]. The SARSCoV-2 virus has made-up off different types of proteins and more than 30,000 nucleotides in its RNA genome. The research on the outer morphology of the proteins confirmed that there are three structural proteins on the surface which comprise small envelop protein (E), spike surface glycoprotein (S) and matrix protein (M). Out of them, $\mathrm{S}$ protein is accountable for more than $80 \%$ of the infections with the cellular membranes [5].

Zinc (Zn)-containing complexes have revealed antiviral properties toward several of viruses according to such corporeal processes such as connection of virus, virus infection, and uncoating [6]. In general, $\mathrm{Zn}$ is an important component that existed in tissues of muscle, brain, skin and bone in human body. It is also a vital component of different enzyme system involved in metabolism and nucleic acid and protein biosynthesis [6]. It is important to note that, $\mathrm{ZnO}$ NPs are more effortlessly absorbed by the body than $\mathrm{Zn}$ itself. ZnONPs are presently utilized in the food industry as preservative and in packaging. According to the US Food and Drug Administration (FDA) ZnO-NPs is safe substance, therefore, ZnO-NPs is impressive for use in biomedical applications. ZnO NPs are known for its low economic cost and less toxicity, therefore, ZnO NPs can be broadly used for various applications such as antibacterial, anticancer, antidiabetic, anti-inflammatory, drug delivery and wound healing [7] 
(Figure 1). Zn-containing complexes were freshly accounted to exhibit anti SARS-CoV-2 activity [8]. Thus, Zn-containing drugs are commonly prescribed in COVID-19 protection protocols.

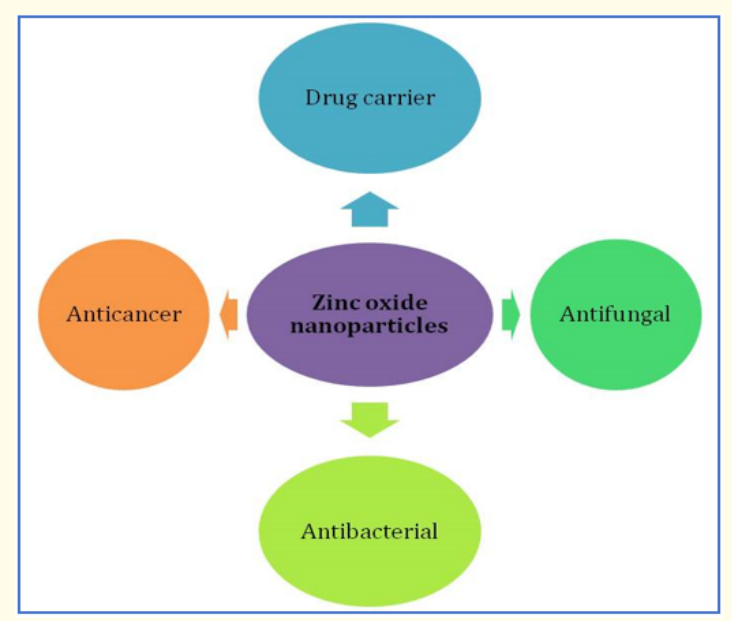

Figure 1: Biomedical applications of zinc oxide nanoparticles.

\section{Why NPs are more effective}

Viruses and NPs have similar size, of about 20-300 $\mathrm{nm}$ for the most studied viruses and 1-100 $\mathrm{nm}$ for NPs [9]. Since viruses have intended strategies to contaminate all living organisms, scientists have been enthused by viruses to design NPs, in particular for targeted drug and gene delivery [10]. In particular, it has been predicted that nanotechnology-based pharmaceutical formulations could be influential to bind the COVID19 pandemic [11-13]. NPs can be tailored for deterrence, diagnosis and treatments of the COVID-19 [14]. With reference to prevention, colloidal mixtures of zinc, silver and copper NPs can be used as disinfectant and gold NPs can be designed for rapid detection of the coronavirus. Likewise, plastics holding antimicrobial silver NPs, graphene or carbon nanotubes offer improved protection to healthcare workers or healthy persons.

\section{NPs based vaccines}

As the international attempt to vaccinate all the individuals worldwide against COVID-19 gathers pace, the demand for vaccine doses that can be used in all countries and climates is massive. Scientists from Chungbuk National University have reported a new vaccine candidate that activates an immune reply using antigens attached to NPs, potentially bypassing the requirement for cold storage during deliverance (Kim., et al. 2021). One of the major challenges in vaccinating the world's population is receiving the vaccine competently to each situate it is desirable. The vaccine in present exercise all need reliable cold storage, and in cases ultra- cold storeroom, to stay efficient. By being make from NPs group that is naturally incredibly thermostable, this latest entrant may not need such conditions (Kim., et al. 2021).

\section{NPs enhanced vaccine immunity}

Several categories of NPs are connected with inherent physical possessions that can trigger an immune reply. Gold, Zn, carbon, dendrimers, polymers and liposome NPs have all been originate to persuade cytokine and antibody responses. These special properties of NPs have therefore increased the effectiveness of NPs from release vehicles for vaccines to adjuvant that can be augmenting the immunogenicity of vaccine contender. NPs that are employed for this reason are recognized as nano-immuno activators or stimulators. Iron, silica and polymeric (chitosan) NPs are some of the good examples of nano-immuno stimulators.

\section{NPs for vaccine delivery}

In contrast to usual vaccine methods, nano carrier-based delivery systems offer various benefits such as, greater defense against premature degradation, high-quality stability, and enhanced adjuvant behavior. In addition to their defensive character, nano-carriers can also recover the specificity of antigen delivery to APCs and augment the period of antigen appearance to these cells and other vital immune cells needed to achieve long-term immunity (Figure 2).

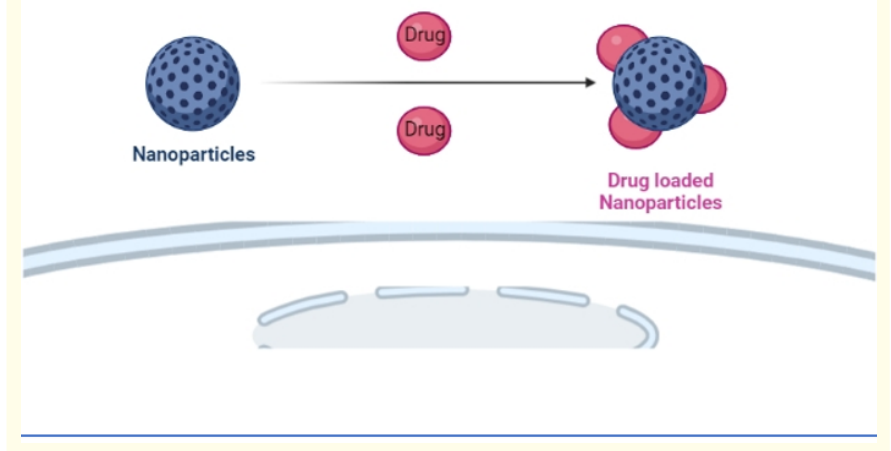

Figure 2: Nanoparticle used for drug delivery.

\section{Conclusion}

ZnO-NPs have exhibited very promising biomedical applications based on their inherent ability to promote reactive oxygen species formation. These specializations of ZnO-NPs make them a perfect antibacterial, drug delivery and antiviral agent (Figure 1 and 2). Although, $\mathrm{ZnO}$ is considered as relatively safe oxide and has been 
permitted by the FDA for cosmetic purposes. However, appliance of ZnO-NPs as vaccine delivery system, as well as immunotherapeutic agents, is still at a pioneer stage of development. Various troubles, such as problem in reproducible large quantity of ZnO-NPs with same characteristics and required properties and a small of basic considerate of the connections of nano-bio interfaces, needs to be determined in upcoming revision.

\section{Conflict of Interest}

The authors declare that they have no financial interest and no conflict of interest.

\section{Bibliography}

1. Itani R., et al. "Optimizing use of theranostic nanoparticles as a life-saving strategy for treating COVID19 patients". Theranostics 10 (2020): 5932-5942.

2. Sivansankarapillai VS., et al. "On facing the SARS-CoV-2 (COVID-19) with combination of nanomaterials and medicine: possible strategies and first challenges". Nanomaterials 10 (2020): E852.

3. Somvanshi S., et al. "Multifunctional nano-magnetic particles assisted viral RNA-extraction protocol for potential detection of COVID-19". Materials Research Innovations 25.3 (2020).

4. Uskokovic V. "Why have nanotechnologies been underutilized in the global uprising against the coronavirus pandemic?" Nanomedicine (Lond) 15 (2020): 1719-1734.

5. Rabiee N., et al. "COVID-19 and picotechnology: potential opportunities". Medical Hypotheses 144 (2020): 109917.

6. Kumar A., et al. "Potential role of zinc supplementation in prophylaxis and treatment of COVID-19". Medical Hypotheses 144 (2020): 109848.

7. Jiang J., et al. "The advancing of zinc oxide nanoparticles for biomedical applications". Bioinorganic Chemistry and Applications (2018).

8. Te Velthuis AJW., et al. " $\mathrm{Zn}^{2+}$ inhibits coronavirus and arterivirus RNA polymerase activity in vitro and zinc ionophores block the replication of these viruses in cell culture". PLoS Pathogen 6 (2010): e1001176.

9. Singh L., et al. "The role of nanotechnology in the treatment of viral infections". Therapeutic Advances in Infectious Disease 4 (2017): 105-131.
10. Wen AM and Steinmetz NF. "Design of virus-based nanomaterials for medicine, biotechnology, and energy". Chemical Society Reviews 45 (2016): 4074-4126.

11. Campos EVR., et al. "How can nanotechnology help to combat COVID-19? Opportunities Urgent Need". Journal of Nanobiotechnology 18 (2020): 125.

12. Khan AH., et al. "COVID-19 transmission, vulnerability, persistence and nanotherapy: a review". Environmental Chemistry Letters 7 (2021): 1-15.

13. Rangayasami A., et al. "Infuence of nanotechnology to combat against COVID-19 for global health emergency: a review". Sensors International 2 (2021).

14. Vahedifard F and Chakravarthy K. "Nanomedicine for COVID-19: the role of nanotechnology in the treatment and diagnosis of COVID-19". Emergency Material 4 (2021): 75-99.

Volume 5 Issue 7 July 2021

(c) All rights are reserved by Mohammad Faizan., et al. 